\title{
HDAC and HDACi: pathogenetic and mechanistic insights
}

Epigentic mechanisms help to regulate proinflammatory cytokine production and, thus, might be important in the pathogenesis of rheumatoid arthritis (RA). Histone deacetylases (HDACs) remove acetyl groups from lysine residues of histones, resulting in chromatin condensation and repression of transcription; as key players in the control of chromatin dynamics they could be a novel therapeutic target in RA. Despite extensive research, many questions remain unanswered regarding the role of HDAC in RA pathogenesis, whether HDAC inhibitors (HDACi) could really work in this disease, and the mechanisms of action of these agents. Two new papers add to our knowledge of this interesting area of research.

Firstly, publishing in Arthritis \& Rheumatism, Michael McDermott and colleagues investigated HDAC activity in peripheral blood mononuclear cells (PBMC) from patients with RA and healthy controls, before and after 12 weeks of therapy with the anti-TNF agent etanercept. HDAC activity was higher in patients with RA than in controls and did not reduce after etanercept therapy. "Dysregulated HDAC activity in PBMC from RA patients is a novel finding and we hypothesize that this may be an important mechanism in perpetuation of RA," explains McDermott.

The authors also tested the effects of two HDACi on cytokine production: trichostatin A (TSA), a nonselective HDACi, and MI192, a new HDAC3-selective inhibitor developed by Ron Grigg. MI192 showed a dose-dependent inhibition of IL-6 in RA PBMC but not in healthy PBMC, whereas TSA was a potent inhibitor of IL- 6 production by both cell types.

In the second paper, published in Annals of the Rheumatic Diseases, Kris Reedquist and coworkers studied the effects of TSA and another HDACi, ITF2357 (which has shown efficacy as a treatment for systemic onset juvenile idiopathic arthritis), on IL-6 production by RA fibroblast-like synoviocytes (RA FLS), to see how these agents regulate gene expression. Both HDACi inhibited the production of IL- 6 by RA FLS after stimulation with
IL-1 $\beta$, TNF or Toll-like receptor ligands. However, adding cyclohexamide (a protein translation inhibitor) did not rescue IL-6 production. "This suggested that we could exclude the idea that HDACi were suppressing IL-6 production via epigenetic mechanisms", explains Reedquist, as this would require the synthesis of a novel repressor protein. By analyzing the kinetics of IL- 6 mRNA induction and degradation, the authors showed that HDACi enhanced IL-6 mRNA degradation.

Reedquist concludes, "This study is the first to provide a tangible, defined molecular mechanism of action as to how HDACi might regulate inflammatory gene expression in RA synovial cells and, by extension, which might be useful in identifying biomarkers if clinical trials are initiated in RA."

Jenny Buckland

Original articles Gillepsie, J. et al. Histone deacetylases are dysregulated in rheumatoid arthritis and a novel HDAC3 selective inhibitor reduces IL- 6 production by PBMC of RA patients. Arthritis Rheum. doi:10.1002/art.33382 | Grabiec, A. M. et al. Histone deacetylase inhibitors suppress rheumatoid arthritis fibroblast-like synoviocyte and macrophage IL- 6 production by accelerating mRNA decay. Ann. Rheum. Dis. doi:10.1136/ard.2011.154211 